\title{
Modeled and Measured Instruction Fetching Performance for Superscalar Microprocessors
}

\author{
Steven Wallace, Member, IEEE, and Nader Bagherzadeh, Senior Member, IEEE
}

\begin{abstract}
Instruction fetching is critical to the performance of a superscalar microprocessor. We develop a mathematical model for three different cache techniques and evaluate its performance both in theory and in simulation using the SPEC95 suite of benchmarks. In all the techniques, the fetching performance is dramatically lower than ideal expectations. To help remedy the situation, we also evaluate its performance using prefetching. Nevertheless, fetching performance is fundamentally limited by control transfers. To solve this problem, we introduce a new fetching mechanism called a dual branch target buffer. The dual branch target buffer enables fetching performance to leap beyond the limitation imposed by conventional methods and achieve a high instruction fetching rate.
\end{abstract}

Index Terms-Computer architecture, instruction fetching, superscalar microprocessor, performance analysis, branch target buffer.

\section{INTRODUCTION}

$\mathrm{T}$ HE goal of a superscalar microprocessor is to execute multiple instructions per cycle. It relies on instructionlevel parallelism (ILP) to achieve this goal [9]. Depending on what type of programs and assumptions are used, researchers have shown that parallelism anywhere from 4 to 90 is available [10], [13], [12], [15]. Unfortunately, all of this potential parallelism will never be utilized if the instructions are not delivered for decoding and execution at a sufficient rate.

The underlying problem in fetching instructions using a control flow architecture is control transfers. Even with perfect branch prediction, conditional, and unconditional branches disrupt the sequential addressing of instructions. The nonsequential accessing of instructions causes difficulty with fetching instructions in hardware. As a result, the instruction fetcher restricts the amount of concurrency available to the processor [14].

In fact, as will be shown in this paper, it can be the greatest factor limiting performance. For example, an eight-way superscalar processor with a simple fetching hardware could only expect to fetch less than four instructions per cycle with programs included in SPECint95. This accounts for over 50 percent of the loss in potential speedup regardless of any other issues. Thus, the performance is severely reduced even if the ILP in the program and execution pipeline would be able to execute eight instructions per cycle.

Branch prediction foretells the outcome of conditional branch instructions. Instruction fetch prediction determines the next instruction to fetch from the memory subsystem [3]. Instruction fetch mechanisms involve the process of how instructions are fetched from memory and delivered to the decoder. This paper focuses on hardware instruction fetching mechanisms. Hence, only instruction fetching

\footnotetext{
- The authors are with the Department of Electrical and Computer Engineering, University of California at Irvine, Irvine, CA 92697.

E-mail: \{swallace; nader\}@ece.uci.edu.
}

Manuscript received 23 June 1995; revised 30 Mar. 1998.

For information on obtaining reprints of this article, please send e-mail to: tpds@computer.org, and reference IEEECS Log Number 104578. performance is evaluated and does not attempt to evaluate any other performance issues (such as branch prediction, cache, execution, etc.). Also, in our study we did not include the effects due to system interference. Our goal is to describe, evaluate, and provide solutions to the first step in a series of hurdles for exploiting high levels of ILP.

Although we will only discuss hardware techniques, we cannot ignore the potential benefit by software techniques. Using software techniques, the probability of a control transfer instruction can be reduced. Loop unrolling is one method. A relatively new technique proposed by Calder and Grunwald is most promising [2]. By rearranging basic blocks, conditional branches become more likely to be "not taken." This means that the probability of a control transfer instruction is reduced because a "not taken" branch is not a control transfer. Nevertheless, software will only be able to make limited improvements, and the hardware techniques presented in this paper will be able to boost instruction fetching performance after software improvements. Furthermore, unlike software techniques, hardware techniques are able to address limitations created by control transfers.

To begin with, we describe our fetching model and the terms we use in our analysis. Then, we show why and how much performance is currently limited by control transfers. Three different cache options are then briefly described: a simple cache type, an extended cache type, and a selfaligned cache type. The way in which prefetching is applied in hardware is described. Next, the dual branch target buffer is described.

The theory behind the fetching techniques gives insight into fetching problems and can give expected performance under given conditions. Therefore, a probabilistic model based on the probability of a control transfer is presented for all combinations of the fetching techniques described. The models are evaluated under several different conditions. To verify that these models predict accurately and to show what real conditions provide, the SPEC95 suite of benchmarks are simulated using the different fetching techniques presented. 


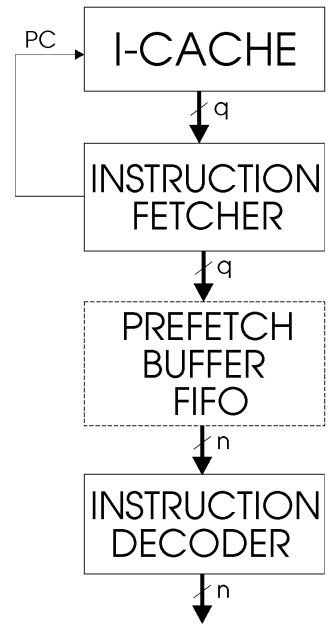

Fig. 1. Fetching block diagram.

\section{Fetching Model}

This section describes the fetching model used in the rest of the paper. The cache line size is defined to be the size of a row in the instruction cache. The terms "line" and "row" are used interchangeably. This determines the maximum number of instructions that can be accessed simultaneously in one cycle. Also, a block is defined to be a group of sequential instructions. A block's width is the maximum number of instructions allowable.

Fig. 1 is a block diagram showing the different fetching steps. The instruction cache reads the requested fetch block of width $q$ and returns it to the instruction fetcher. The instruction decoder receives a decode block of width $n$. If prefetching is applied, up to $q$ new instructions from the instruction fetcher go into the prefetch buffer FIFO queue and $n$ instructions come out. This implies $q>n$ in the diagram. Otherwise, if prefetching is not used, the fetch and decode widths are equal, and the instruction fetcher delivers instructions directly to the decoder.

The instruction fetcher is responsible for determining the new starting PC each cycle and sending it to the instruction cache. It cooperates with a branch predictor or branch target buffer, if employed. Calder and Grunwald [1] describe different techniques for fast PC calculation. Whichever technique is used, the new PC must be determined in the same cycle. Also, after the instruction fetcher receives the fetch block from the instruction cache, it performs preliminary decoding to determine the instruction type (or uses prediction/predecoding methods). Instructions after the first instruction that transfers control are invalidated.

Johnson defines an instruction run to be the sequentially fetched instructions between branches [9]. In this paper, an instruction run is further specified to be between instructions that transfer control. A control transfer instruction includes unconditional jumps and calls, conditional branches that are taken, and any other instruction that transfers control, such as a trap. The run length is the number of instructions in a run. In addition, a block run is defined to be the instructions from the start of the block to the end of the block or the first instruction that transfers control. The block run length is the number of instructions in a block run.

\section{Fetching Limitation}

Let $n$ be the width of a block and $b$ be the probability that an instruction transfers control. The expected block run length, $r(n, b)$, is

$$
r(n, b)=n(1-b)^{n}+\sum_{i=1}^{n} i(1-b)^{i-1} b=\frac{1-(1-b)^{n}}{b} .
$$

Equation (1) represents the weighted sum of all events that could occur in a sequence of $n$ instructions. The first term is the case where there is no control transfer in a block. The second term represents all possible permutations of a control transfer in a block. The limit of $r(n, b)$ as the block width increases is given by

$$
\lim _{n \rightarrow \infty} r(n, b)=\frac{1}{b} .
$$

If a control transfer requires another cycle to reach the target address, then only one block of instructions can be fetched in a cycle. Regardless of the type of software scheduling or hardware techniques used to improve fetching, $1 / b$ is the limit for the average number of instructions fetched per cycle. Under these conditions, $1 / b$ is the maximum average number of instructions per cycle that can be executed on any single-threaded control-flow architecture.

Here is an example to illustrate this fundamental fetching limitation. Suppose a program executes a million instructions, and one hundred thousand of these instructions transfer control. The probability of a control transfer instruction is therefore one tenth, and an average of 10 instructions fetched per cycle is the theoretical limit. Since each control transfer instruction requires one cycle, to execute this program would require a minimum of a hundred thousand cycles.

\section{Hardware TechNiques}

This section describes hardware techniques which perform instruction fetching. To begin with, three cache types are described: a simple cache, an extended cache, and a selfaligned cache. Next, prefetching is described. Finally, a new mechanism to fetch two blocks per cycle, a dual branch target buffer, is introduced.

\subsection{Simple Cache}

A straightforward approach to fetch instructions from the instruction cache is to have the line size equal the width of the fetch block. If the starting PC address is not the first position in the corresponding row of the instruction cache, then the appropriate instructions are invalidated and fewer than the fetch width are returned. As with all fetching techniques, if there is an instruction that transfers control, instructions after it are invalidated.

Fig. 2 shows an example for the simple fetching mechanism. In this example, the second instruction in the first block is a taken branch, so the third and fourth instructions are invalidated. Also, only two instructions from the second block are valid. Altogether, only four out of a potential eight instructions are used for instruction decoding and execution, which illustrates the problem with this simple approach. 


\section{Starting PC}

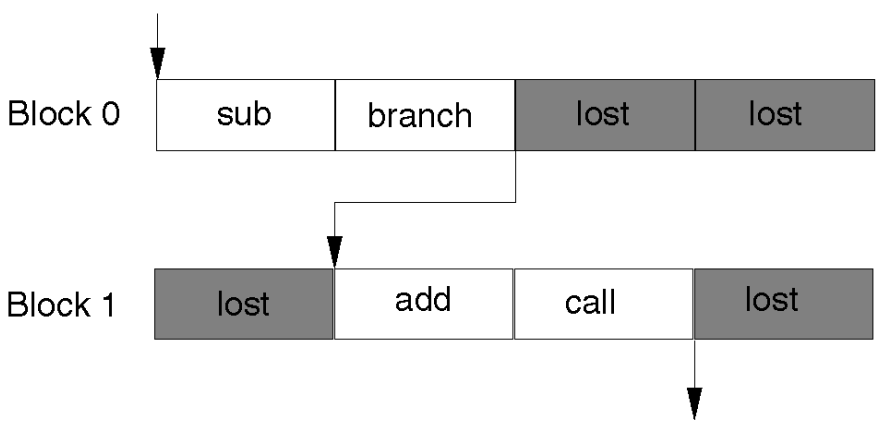

Fig. 2. Simple fetching example.

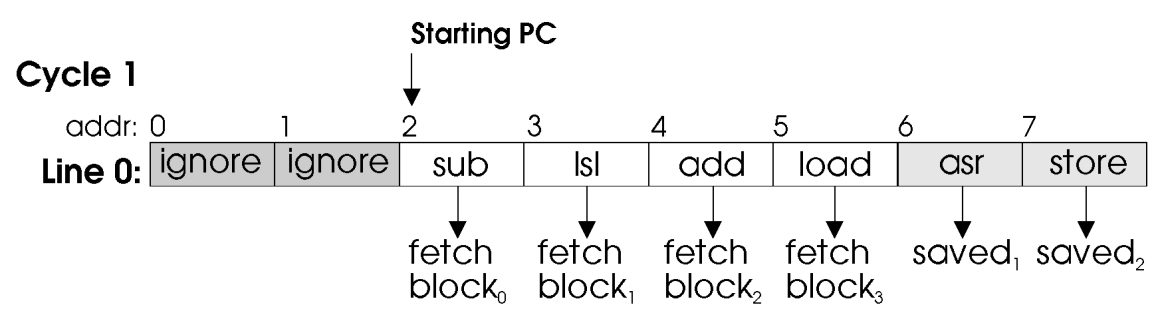

Cycle 2

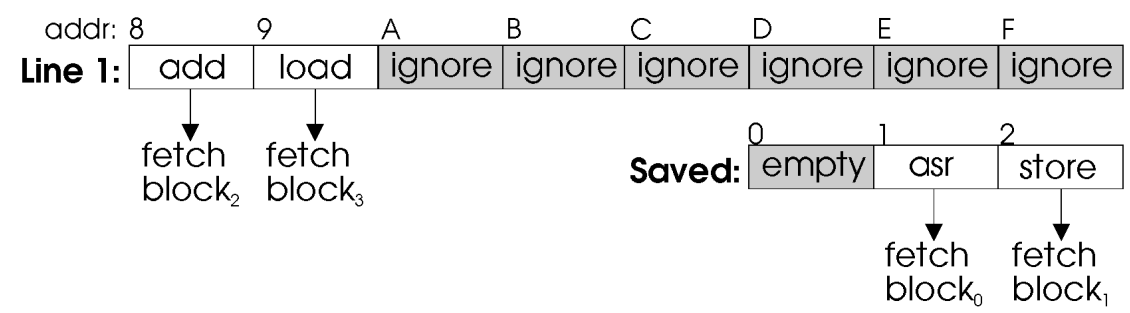

Fig. 3. Extended fetching example.

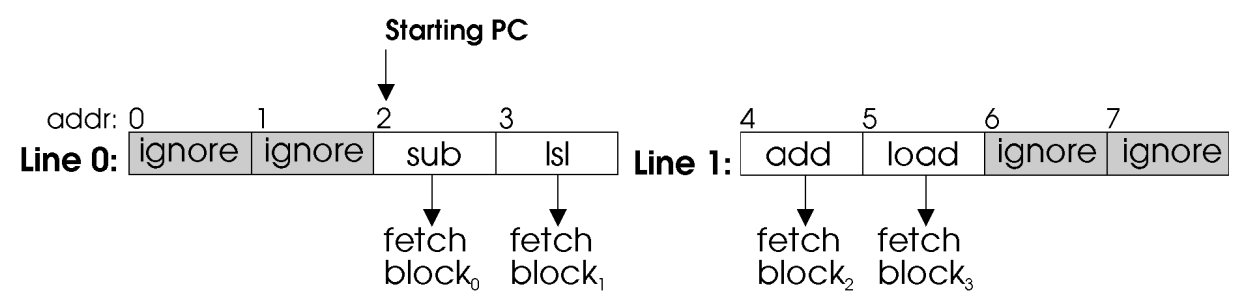

Fig. 4. Self-aligned fetching example.

\subsection{Extended Cache}

One way to reduce the chance that instructions will be lost from an unaligned target address of a control transfer instruction is to extend the instruction cache line size beyond the width of the fetch block. To avoid lost instructions on sequential reads that are not block aligned, the instruction fetcher must be able to save the last $n-1$ instructions in a row and combine them with instructions that are read the next cycle. Only when there is a control transfer to the last $n-1$ instructions in a cache row are instructions lost due to an unaligned target address.

Fig. 3 is an example of the extended cache fetching technique using $n=4$ and an extended cache line size of eight instructions. The starting PC in this example is at the third instruction in Line 0. Four instructions are returned to the instruction fetcher in Cycle 1. The last two instructions in Line 0 are saved for the next cycle. During Cycle 2, the instruction fetcher combines two new instructions read from
Line 1 and the two instructions saved the previous cycle. There is no need to save any instructions this cycle because the line can be reread and still be able to return four instructions.

\subsection{Self-Aligned Cache}

The target alignment problem can be solved completely in hardware with a self-aligned instruction cache. The instruction cache reads and concatenates two consecutive rows within one cycle so as to always be able to return $n$ instructions. To implement a self-aligned cache, the hardware must either use a dual-port instruction cache, perform two separate cache accesses in a single cycle, or split the instruction cache into two banks. Using a two-way interleaved (i.e., two banks) instruction cache is preferred for both space and timing reasons [5], [8], [7].

Fig. 4 is an example of the self-aligned cache fetching technique using $n=4$. Only the last two instructions in Line 0 are available for use because the starting PC is not at the 


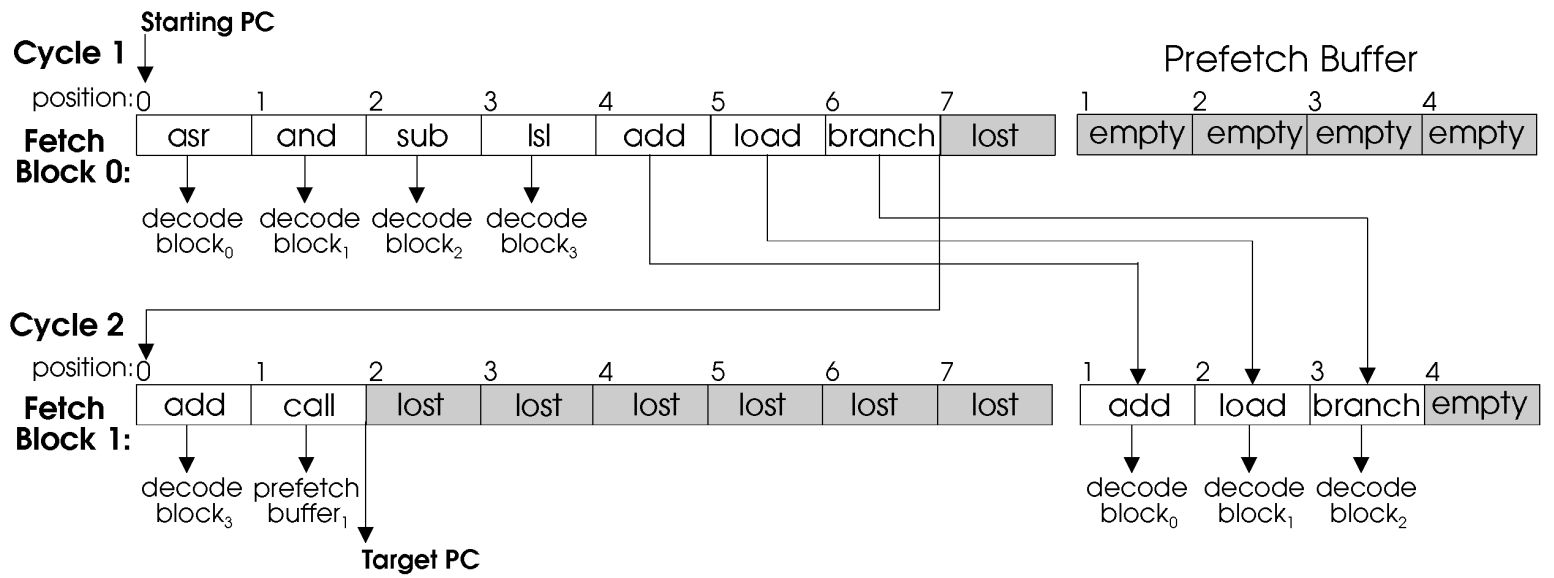

Fig. 5. Prefetch example.

first position. Since the following line is read and available during the same cycle, four instructions are returned by combining the two instructions from Line 0 and the first two instructions from Line 1.

\subsection{Prefetching}

All of the above cache types can be used in conjunction with prefetching. Prefetching helps improve fetching performance, but fetching is still limited because instructions after a control transfer must be invalidated.

The fetch width, $q, q \geq n$, is the number of instructions that are examined for a control transfer. Let $p$ be the size of the prefetch buffer. After the instruction fetcher searches up to $q$ instructions for a control transfer, valid instructions are stored into a prefetch buffer. Each cycle, the instruction decoder removes the oldest $n$ instructions from the prefetch buffer. In essence, the prefetch buffer enables an average performance closer to the larger expected run length of $q$ instructions compared to $n$ instructions.

Fig. 5 shows an example using prefetching with $n=4$, $q=8$, and $p=4$. Starting with an empty prefetch buffer, there are seven valid instructions (this example shows a complete block of $q=8$ instructions returned by the instruction cache to the instruction fetcher) before branch. Four instructions are used in this cycle, while the remaining three valid instructions are put in the prefetch buffer for later use. In the next cycle, a block of instructions is read starting with the target address of the branch. Only two instructions are valid because a call instruction was detected. As a result, three instructions from the buffer and the first add instruction are used, while the remaining call instruction is put into the prefetch buffer.

\subsection{Dual Branch Target Buffer}

In this section, the dual branch target buffer (DBTB) is introduced. It is based on the original branch target buffer (BTB) design by Lee and Smith [11]. Unlike the previous techniques mentioned thus far, the DBTB can bypass the limitation imposed by a control transfer. The DBTB is similar to the Branch Address Cache introduced by Yeh et al. [16], except the DBTB does not grow exponentially. Conte et al. introduced the collapsing buffer, which allows intrablock branches [5]. The DBTB can handle both intrablock and interblock branches.
The purpose of a BTB is to predict the target address of the next instruction given the address of the current instruction. This idea is taken one step further. Given the current $P C$, the DBTB predicts the starting address of the following two lines, which overcomes the limitation of a single prediction of the BTB. Using the predicted addresses for the next two lines, a dual-ported instruction cache is used to simultaneously read them. Hence, the first line may have a control transfer without requiring another cycle to fetch the subsequent line.

The DBTB is indexed by the starting address of the last row currently being accessed in the instruction cache (i.e., the current PC). The entry read from the DBTB can be viewed as two BTB entries, BTB1 and BTB2. The DBTB entry indexed may match both in BTB1 and BTB2, in one or the other, or none at all. This allows a single DBTB entry to be shared between two different source PCs. Although physically they are one entry, logically they are separate.

Fig. 6 is a block diagram of a DBTB entry and shows how it is used in determining the following two rows' PC starting address, PC1 and PC2. The tag of the current PC is checked against the PC tag found in BTB1. If it matches, then the predicted PC1 found in BTB1 is used. Otherwise, the prediction is to follow through to the next row of the instruction cache. If the value predicted for PC1 matches the value in BTB2, then the prediction for PC2 in BTB2 is used; else, PC2 is predicted to be the next row after PC1. The exit position in a DBTB entry indicates where the control transfer (or follow through) is predicted to occur. The DBTB entry also contains branch prediction information about all the potential branches in the referenced line. It may contain no information at all, a one-bit prediction, a two-bit saturating prediction, or information for other branch prediction mechanisms.

To save space, an alternative design of the DBTB would be to logically unify BTB1 and BTB2. A block diagram is shown in Fig. 7. Only one PC source can be valid, so only one PC tag would now need to be stored. In addition, for space savings, the time it takes for PC2 to be ready is reduced because the predicted PC1 does not need to be checked against the tagged PC1 in BTB2. As a result, the logically unified DBTB's critical path is the same as a standard BTB. This improvement may be critical in a processor's cycle time. The 


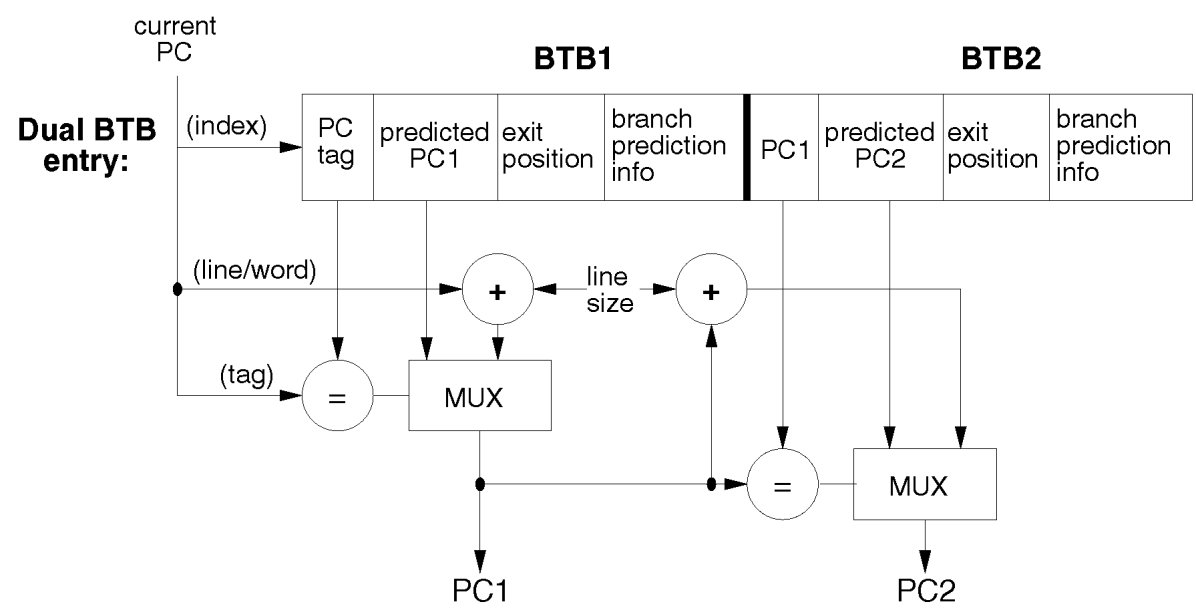

Fig. 6. Block diagram of dual branch target buffer entry.

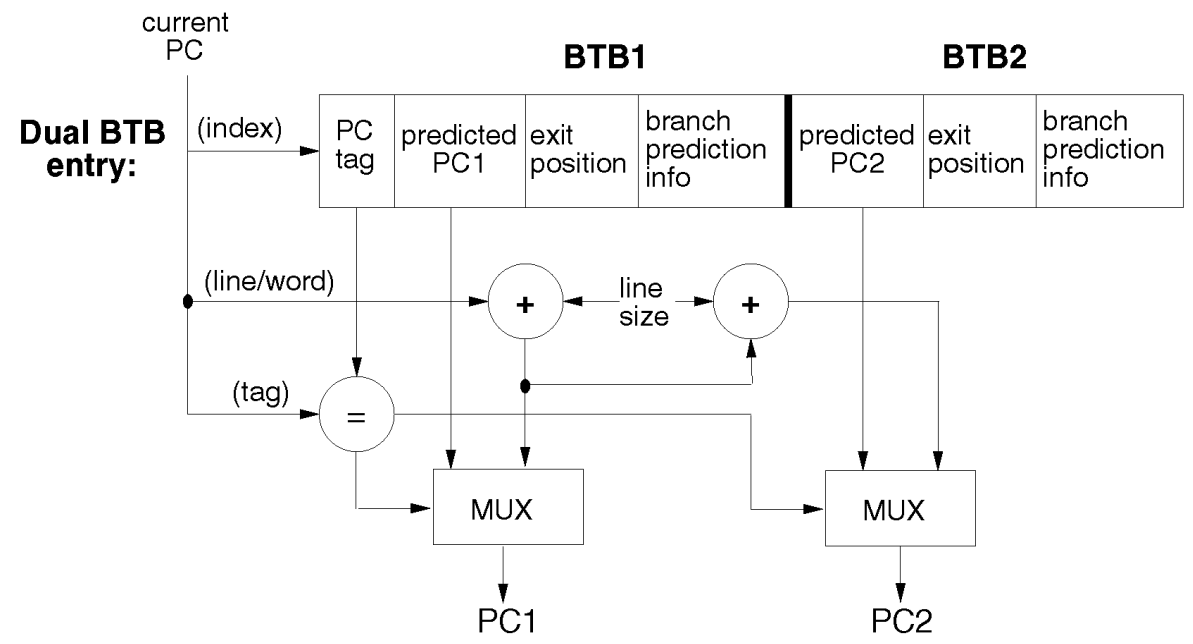

Fig. 7. Logically unified dual branch target buffer entry.

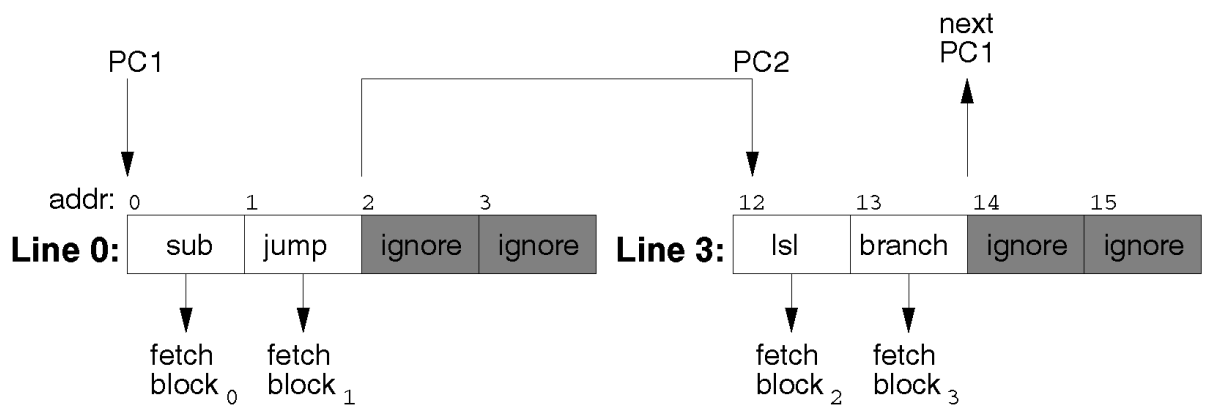

Fig. 8. Dual branch target buffer example.

drawback is BTB2 must be invalidated to reflect a followthrough prediction when BTB1 is updated, which can reduce accuracy of prediction. On the other hand, a BTB2 misprediction does not need to invalidate BTB1.

The DBTB has many different configurations, many similar to the traditional BTB. Its options include the number of entries, associativity, branch prediction, and a one or two tagged system. A DBTB can be used with a simple, extended, or self-aligned cache, and with or without prefetching. Fig. 8 is a fetching example without prefetching using the DBTB. The previous cycle, BTB1, predicted PC1 to be at Address 0 , and BTB2 predicted Line 0 to exit at position 1 to
PC2 at Address 12. While Line 0 and Line 3 are being read, PC2 is used to index into the DBTB to predict the next PC1 and PC2. Although Line 0 has a jump, a full fetch block of four instructions is returned.

\section{EXPECTEd INSTRUCTION FETCH}

A mathematical model for each type of fetching mechanism from the previous section is presented in this section. The model allows the expected instruction fetching performance to be calculated. This accounts for invalid instructions, but not for mispredicted instructions. The fetching bandwidth is 
only considered, and not other effects from a processor's pipeline. In the next section, the expected performance from this model will be compared with results from simulation.

\subsection{Simple Cache}

Let $L_{i}$ be the probability a control transfer occurs at position $i$, and $E_{i}$ be the probability the starting address in the block is at position $i$. Upon a control transfer, if the target address is equally likely to enter any position in a block, then

$$
\begin{gathered}
E_{1}^{\text {simple }}(n, b)=1-\frac{n-1}{n} c^{\text {simple }}(n, b) \\
E_{i}^{\text {simple }}(n, b)=\frac{c^{\text {simple }}(n, b)}{n}, 2 \leq i \leq n, \\
L_{i}^{\text {simple }}(n, b)=\sum_{j=1}^{i} b(1-b)^{i-j} E_{j}^{\text {simple }}(n, b),
\end{gathered}
$$

where $c(n, b)$ is the probability of a control transfer in a block,

$$
c^{\text {simple }}(n, b)=\sum_{i=1}^{n} L_{i}^{\text {simple }}(n, b)=\frac{n}{\frac{1}{b}+n-1} .
$$

The total expected instructions fetched per cycle for simple fetching is

$$
\begin{aligned}
F^{\text {simple }}(n, b) & =\sum_{i=1}^{n} E_{i}^{\text {simple }}(n, b) r(i, b) \\
& =\frac{c^{\text {simple }}(n, b)}{b}=\frac{n}{1+b(n-1)} .
\end{aligned}
$$

Equation (6) is the weighted sum of the expected number of instructions at each possible starting position.

\subsection{Extended Cache}

The probability the starting address in the block is at position $i$ for the extended cache is

$$
\begin{aligned}
& E_{1}^{\text {extend }}(n, b, m)=1-\frac{m-1}{m} c^{\text {extend }}(n, b, m) \\
& E_{i}^{\text {extend }}(n, b, m)=\frac{c^{\text {extend }}(n, b, m)}{m}, 2 \leq i \leq m .
\end{aligned}
$$

The probability of a control transfer in a block for the extended cache, given the extended cache line size $m, m \geq n$, is

$$
c^{\text {extend }}(n, b, m)=\frac{m-n}{m}\left(1-(1-b)^{n}\right)+\frac{n}{m} \frac{n b}{(1+b(n-1))} .
$$

The expected instructions fetched per cycle is

$$
\begin{aligned}
F^{\text {extend }}(n, b, m) & =\frac{m-n}{m} r(n, b)+\frac{n}{m} F^{\text {simple }}(n, b) \\
& =\frac{(m-n)}{m} \frac{\left(1-(1-b)^{n}\right)}{b}+\frac{n}{m} \frac{n}{(1+b(n-1))} .
\end{aligned}
$$

With the cache line size extended beyond the desired $n$ instructions, if there is a control transfer, $n$ out of $m$ times it is expected to transfer into the last $n$ instructions of the block, which behave as the simple fetching case where less than $n$ instructions are available. The rest of the time, $n$ instructions will be available.

\subsection{Self-Aligned Cache}

The probability of a control transfer in a block for the selfaligned cache is

$$
c^{\text {align }}(n, b)=1-(1-b)^{n} .
$$

The expected instructions fetched per cycle for the selfaligned cache is the expected block run length of width $n$,

$$
F^{\text {align }}(n, b)=r(n, b)=\frac{1-(1-b)^{n}}{b},
$$

because $n$ instructions will always be read from the instruction cache.

\subsection{Prefetching}

All three cache techniques can be used in combination with prefetching. The fetch and decode widths are not equal with prefetching. As a result, $q$, the fetch width, may now be substituted for $n$, the decode width, as a parameter to some of the equations previously defined that did not use prefetching, as will be indicated.

Let $I_{i}^{\text {type }}$ be the probability exactly $i$ instructions are available up to and including a control transfer instruction or the end of the block, where type is one of the three different cache types: simple, extend, or align. The equations for the three types are:

$$
\begin{gathered}
I_{i}^{\text {simple }}(1-b)^{i-1} E_{q-i+1}^{\text {simple }}(q, b)+\sum_{j=1}^{q-i} b(1-b)^{i-1} E_{j}^{\text {simple }}(q, b), \\
I_{i}^{\text {extend }}= \begin{cases}(1-b)^{i-1} E_{m-i+1}^{\text {extend }}(q, b, m) \\
+(1-b)^{i-1} b \sum_{j=1}^{m-i} E_{j}^{\text {extend }}(q, b, m) & 1 \leq i \leq q-1 \\
(1-b)^{q-1} \sum_{j=1}^{m-q+1} E_{j}^{\text {extend }}(q, b, m) & i=q \\
0 & \text { otherwise, }\end{cases} \\
I_{i}^{\text {align }}= \begin{cases}(1-b)^{i-1} b & 1 \leq i \leq q-1 \\
(1-b)^{q-1} & i=q \\
0 & \text { otherwise. }\end{cases}
\end{gathered}
$$

Let $P_{i}$ be the probability the prefetch buffer contains $i$ instructions. Fig. 9 illustrates the transition from one buffer state to another. It does not show all possible transitions. The prefetch buffer increases in size when the number of new instructions is greater than $n$. It will remain in the same state if exactly $n$ new instructions are available. It decreases in size when fewer than $n$ new instructions are available. The zero and full boundary states have additional possible transitions.

The probability the prefetch buffer is in state $i$ is $P_{i}^{\text {type }}=$

$$
\left\{\begin{array}{llll}
\sum_{j+k \leq n} P_{j}^{\text {type }} I_{k}^{\text {type }} & i=0 & 0 \leq j \leq p & 1 \leq k \leq q \\
\sum_{j+k=n+i} P_{j}^{\text {type }} I_{k}^{\text {type }} & 1 \leq i \leq p-1 & 0 \leq j \leq p & 1 \leq k \leq q \\
\sum_{j+k \geq n+p} P_{j}^{\text {type }} I_{k}^{\text {type }} & i=p & 0 \leq j \leq p & 1 \leq k \leq q .
\end{array}\right.
$$

Also, $\sum_{i=0}^{p} P_{i}=1$. Equation (15) can be expanded as a system of linear equations and solved for each $P_{i}$. 


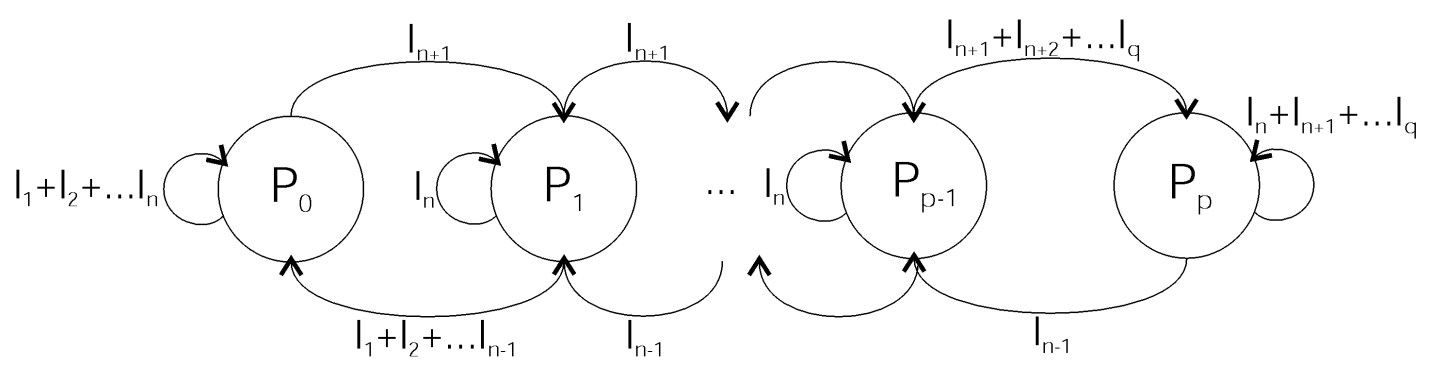

Fig. 9. Prefetch buffer state diagram.

TABLE 1

INSTRUCTIONS FETCHED PER CYCLE $(n=4)$

\begin{tabular}{|r|r|rr|rr|rr|}
\hline Program & $1 / b$ & \multicolumn{2}{|c|}{ simple } & \multicolumn{2}{c|}{ extend } & \multicolumn{2}{c|}{ align } \\
& & pred & obs & pred & obs & pred & obs \\
\hline go & 11.6 & 3.18 & 3.20 & 3.35 & 3.34 & 3.51 & 3.56 \\
gcc & 7.5 & 2.86 & 2.86 & 3.07 & 3.06 & 3.27 & 3.41 \\
m88ksim & 10.2 & 3.09 & 3.01 & 3.27 & 3.12 & 3.45 & 3.48 \\
compress & 9.93 & 3.07 & 3.31 & 3.25 & 3.43 & 3.44 & 3.59 \\
li & 6.9 & 2.78 & 2.75 & 2.99 & 3.10 & 3.21 & 3.31 \\
ijpeg & 21.5 & 3.51 & 3.51 & 3.62 & 3.59 & 3.73 & 3.73 \\
perl & 7.8 & 2.89 & 2.88 & 3.09 & 3.14 & 3.29 & 3.36 \\
vortex & 9.2 & 3.01 & 2.90 & 3.20 & 3.02 & 3.39 & 3.57 \\
\hline tomcatv & 22.0 & 3.52 & 3.40 & 3.63 & 3.47 & 3.74 & 3.69 \\
swim & 114 & 3.90 & 3.86 & 3.92 & 3.93 & 3.95 & 3.96 \\
su2cor & 11.7 & 3.19 & 3.25 & 3.35 & 3.40 & 3.52 & 3.62 \\
hydro2d & 12.9 & 3.24 & 3.24 & 3.40 & 3.34 & 3.56 & 3.63 \\
mgrid & 79.6 & 3.85 & 3.68 & 3.89 & 3.81 & 3.93 & 3.85 \\
applu & 25.3 & 3.58 & 3.45 & 3.67 & 3.61 & 3.77 & 3.73 \\
turb3d & 14.6 & 3.32 & 3.37 & 3.46 & 3.46 & 3.61 & 3.69 \\
apsi & 54.9 & 3.79 & 3.76 & 3.84 & 3.81 & 3.89 & 3.87 \\
fpppp & 13.6 & 3.28 & 3.13 & 3.43 & 3.30 & 3.58 & 3.60 \\
wave5 & 23.6 & 3.55 & 3.54 & 3.65 & 3.62 & 3.75 & 3.74 \\
\hline
\end{tabular}

The total expected instruction fetch for each of the different cache types with prefetching is

$$
F_{\text {prefetch }}^{\text {type }}(p, q, n, b)=n-\sum_{i=1}^{n-1}(n-1) \sum_{\substack{j+k=i \\ 0 \leq j \leq p \\ 1 \leq k \leq q}} P_{j}^{\text {type }} I_{k}^{\text {type }} .
$$

Notice (16) depends only on the last $n-2$ prefetch buffer state sizes since if there are $n-1$ or more instructions in the prefetch buffer, $n$ instructions are guaranteed for that cycle.

A problem can arise with prefetching and simple cache type. The prefetch buffer can be full and instructions from the fetch block go unused. If this happens, the starting address of the next cycle will not be the first position, so $q$ instructions will not be available. Therefore, (3) needs to be modified to include this effect, unless a hardware solution similar to that of the extended cache is included. The hardware would need to save instructions left over on a prefetch buffer overflow for the following cycle. If this is done, (12) is an accurate model.

\subsection{Dual Block Fetching}

Fetching two blocks per cycle (via the DBTB) with the simple, extended, or self-aligned cache without prefetching is simply twice the expected value for half the block size,
TABLE 2

Instructions FetChed PER CYCLE With PREFETCHing $(n=4)$

\begin{tabular}{|r|r|rr|rr|rr|}
\hline Program & $1 / b$ & \multicolumn{2}{|c|}{ simple } & \multicolumn{2}{c|}{ extend } & \multicolumn{2}{c|}{ align } \\
& & pred & obs & pred & obs & pred & obs \\
\hline go & 11.6 & 3.95 & 3.93 & 3.95 & 3.97 & 3.99 & 4.00 \\
gcc & 7.5 & 3.76 & 3.62 & 3.91 & 3.77 & 3.96 & 3.99 \\
m88ksim & 10.2 & 3.92 & 3.89 & 3.97 & 3.95 & 3.99 & 4.00 \\
compress & 9.93 & 3.91 & 3.92 & 3.97 & 3.98 & 3.99 & 3.99 \\
li & 6.9 & 3.69 & 3.71 & 3.87 & 3.87 & 3.94 & 3.98 \\
ijpeg & 21.5 & 3.99 & 3.96 & 4.00 & 3.96 & 4.00 & 4.00 \\
perl & 7.8 & 3.79 & 3.66 & 3.92 & 3.80 & 3.97 & 3.99 \\
vortex & 9.2 & 3.88 & 3.58 & 3.96 & 3.68 & 3.98 & 4.00 \\
\hline tomcatv & 22.0 & 3.99 & 3.95 & 4.00 & 3.99 & 4.00 & 4.00 \\
swim & 114 & 4.00 & 4.00 & 4.00 & 4.00 & 4.00 & 4.00 \\
su2cor & 11.7 & 3.95 & 3.86 & 3.99 & 3.99 & 3.99 & 4.00 \\
hydro2d & 12.9 & 3.97 & 3.72 & 3.99 & 3.92 & 4.00 & 4.00 \\
mgrid & 79.6 & 4.00 & 4.00 & 4.00 & 4.00 & 4.00 & 4.00 \\
applu & 25.3 & 4.00 & 4.00 & 4.00 & 4.00 & 4.00 & 4.00 \\
turb3d & 14.6 & 3.98 & 3.69 & 3.99 & 3.77 & 4.00 & 3.99 \\
apsi & 54.9 & 4.00 & 4.00 & 4.00 & 4.00 & 4.00 & 4.00 \\
fpppp & 13.6 & 3.97 & 3.74 & 3.99 & 3.81 & 4.00 & 3.99 \\
wave5 & 23.6 & 3.99 & 3.96 & 4.00 & 3.99 & 4.00 & 4.00 \\
\hline
\end{tabular}

$$
F^{d b t b, t y p e}(n, b)=2 F^{t y p e}\left(\frac{n}{2}, b\right) .
$$

If prefetching is used with dual block fetching, the equation for $I_{k}^{\text {type }}$ in (15) and (16) is replaced with

$$
I_{k}^{d b t b, \text { type }}(q, b)=\sum_{j=0}^{k} I_{j}^{\text {type }}\left(\frac{q}{2}, b\right) I_{k-j}^{\text {type }}\left(\frac{q}{2}, b\right) .
$$

\section{Results ANd Discussion}

This section compares the expected instruction fetch with the actual performance of simulations from the SPEC95 benchmark suite running on the SPARC architecture [6]. The suite was compiled using the SunPro compiler with standard optimizations (-O). Programs were simulated using the Shade instruction-set simulator [4] and ran until completion or the first four billion instructions.

Table 1 shows the predicted and observed instruction fetch count results of these programs using the three cache techniques without prefetching $(n=4)$. Table 2 and Table 3 show the predicted and observed instruction fetch count results using the three cache techniques with prefetching ( $n=4, q=8, p=8$; and $n=8, q=16, p=16$, respectively). 
TABLE 3

Instructions Fetched per CYCle With PRefetChing $(n=8)$

\begin{tabular}{|r|r|rc|cc|cc|}
\hline Program & $1 / b$ & \multicolumn{2}{|c|}{ simple } & \multicolumn{2}{|c|}{ extend } & \multicolumn{2}{|c|}{ align } \\
& & pred & obs & pred & obs & pred & obs \\
\hline go & 11.6 & 6.75 & 6.75 & 7.33 & 7.33 & 7.65 & 7.75 \\
gcc & 7.5 & 5.32 & 5.10 & 5.98 & 5.58 & 6.55 & 6.52 \\
m88ksim & 10.2 & 6.36 & 6.22 & 7.02 & 6.85 & 7.44 & 7.44 \\
compress & 9.93 & 6.27 & 7.19 & 6.94 & 7.45 & 7.38 & 7.64 \\
li & 6.9 & 5.03 & 4.89 & 5.66 & 5.86 & 6.22 & 6.49 \\
ijpeg & 21.5 & 7.79 & 7.14 & 7.93 & 7.51 & 7.97 & 7.89 \\
perl & 7.8 & 5.45 & 5.16 & 6.13 & 5.65 & 6.69 & 6.64 \\
vortex & 9.2 & 6.02 & 5.38 & 6.71 & 5.81 & 7.20 & 7.05 \\
\hline tomcatv & 22.0 & 7.56 & 7.03 & 7.83 & 7.51 & 7.92 & 7.82 \\
swim & 114 & 8.00 & 7.95 & 8.00 & 7.98 & 8.00 & 7.99 \\
su2cor & 11.7 & 6.77 & 6.10 & 7.35 & 6.61 & 7.66 & 7.53 \\
hydro2d & 12.9 & 7.03 & 6.39 & 7.53 & 6.80 & 7.77 & 7.25 \\
mgrid & 79.6 & 8.00 & 7.97 & 8.00 & 7.99 & 8.00 & 8.00 \\
applu & 25.3 & 7.88 & 7.56 & 7.96 & 7.72 & 7.98 & 7.96 \\
turb3d & 14.6 & 7.30 & 5.87 & 7.69 & 6.29 & 7.85 & 6.92 \\
apsi & 54.9 & 7.99 & 7.93 & 8.00 & 7.98 & 8.00 & 8.00 \\
fpppp & 13.6 & 7.15 & 6.09 & 7.60 & 6.43 & 7.80 & 6.96 \\
wave5 & 23.6 & 7.85 & 7.42 & 7.95 & 7.73 & 7.98 & 7.92 \\
\hline
\end{tabular}

The first column in both tables show the value observed for $1 / b$, the average run length. The average dynamic run length of a program is the total number of instructions executed divided by the number of instructions that transferred control. The observed value of $b$ for each program was used in its calculation of the expected fetch.

A concern with the fetching model presented is that the distribution of run lengths is expected to be uniform, but in observing actual program behavior, the distribution is not uniform. It does, however, generally follow the expected distribution. When the expected fetch is calculated via a weighted sum, the outcome is reasonably accurate. As can be seen in the tables, the difference between the predicted and observed fetch count is usually within a few percent.

The expected and observed performance for dual block fetching without prefetching is exactly twice the values listed in Table 1 for $n=8$. Table 4 lists the performance of SPEC95 for dual block fetching with prefetching $(n=8, q=16, p=8)$. The instructions fetched per cycle (IFPC) is listed, as well as the instructions per fetch block (IPB). The results show that close to ideal $(n=8)$ fetching rate is possible, when a two-block fetching mechanism, such as the dual branch target buffer, is used with extended or self-aligned cache and prefetching. In this case, the fetching hardware mechanism no longer restricts instruction fetching, and therefore, the possibility of exploiting instruction-level parallelism and a high instructions per cycle execution rate.

Using a 256-entry, direct-mapped, two-tagged DBTB, we observed that the miss rate was between 10 percent and 20 percent for most of the SPEC95 benchmarks. Also, the miss rates for BTB2 were usually slightly higher than BTB1. BTB1 and BTB2 each behaved similarly to a standard BTB. Although perfect branch accuracy was assumed in Table 4 (to make a fair comparison to the other data), it is important
TABLE 4

IPB AND IFPC FOR DUAL BLOCK FETCHING WITH PREFETCHING

\begin{tabular}{|r|r|rr|rr|rr|}
\hline Program & $1 / b$ & \multicolumn{2}{|c|}{ simple } & \multicolumn{2}{c|}{ extend } & \multicolumn{2}{c|}{ align } \\
& & IPB & IFPC & IPB & IFPC & IPB & IFPC \\
\hline go & 11.6 & 9.90 & 7.79 & 11.2 & 7.90 & 12.3 & 7.98 \\
gcc & 7.5 & 8.01 & 7.18 & 9.3 & 7.49 & 10.5 & 7.93 \\
m88ksim & 10.2 & 9.24 & 7.68 & 11.0 & 7.87 & 11.7 & 7.98 \\
compress & 9.93 & 9.98 & 7.78 & 10.5 & 7.86 & 11.8 & 7.95 \\
li & 6.9 & 7.64 & 7.37 & 9.8 & 7.74 & 10.4 & 7.91 \\
ijpeg & 21.5 & 12.0 & 7.89 & 12.8 & 7.91 & 13.6 & 8.00 \\
perl & 7.8 & 8.37 & 7.36 & 9.9 & 7.67 & 10.7 & 7.93 \\
vortex & 9.2 & 8.07 & 7.14 & 10.6 & 7.52 & 11.8 & 7.99 \\
\hline tomcatv & 22.0 & 11.7 & 7.88 & 12.4 & 7.97 & 13.9 & 8.00 \\
swim & 114 & 15.0 & 7.99 & 15.3 & 8.00 & 15.8 & 8.00 \\
su2cor & 11.7 & 9.5 & 7.53 & 11.2 & 7.78 & 12.4 & 7.99 \\
hydro2d & 12.9 & 9.8 & 7.36 & 11.5 & 7.90 & 12.7 & 8.00 \\
mgrid & 79.6 & 15.5 & 8.00 & 15.6 & 8.00 & 15.7 & 8.00 \\
applu & 25.3 & 12.4 & 7.97 & 13.2 & 8.00 & 13.9 & 8.00 \\
turb3d & 14.6 & 10.6 & 7.38 & 11.9 & 7.53 & 12.5 & 7.94 \\
apsi & 54.9 & 14.0 & 7.98 & 14.7 & 8.00 & 15.1 & 8.00 \\
fpppp & 13.6 & 13.0 & 7.79 & 13.5 & 7.92 & 14.9 & 7.99 \\
wave5 & 23.6 & 12.4 & 7.89 & 13.2 & 7.96 & 14.0 & 7.99 \\
\hline
\end{tabular}

to realize that accurate branch prediction becomes critical since more branches need to be predicted accurately per fetch block.

The overall performance will be much lower than the fetching rates shown when branch prediction, cache misses, execution, etc., of a real microprocessor are simulated. In addition, the difference between the values will be much smaller. These facts do not devalue the results presented. These results show the upper limit achievable using different fetching mechanisms presented, both in theory and in simulation. No doubt, as branch prediction accuracy, cache performance, and execution performance continue to improve in the future, the demand for adequate fetching mechanisms will increase.

\section{CONCLUSION}

Many programs have sufficient ILP to execute eight instructions per cycle and a superscalar microprocessor can be designed to decode and execute eight instructions per cycle. Unfortunately, because of control transfers in programs, a simple fetching mechanism cannot reach this high demand. In fact, it falls far short.

The extended and self-aligned cache techniques also showed extremely poor instruction fetching performance, although the self-aligned cache always performed better than the other two. Prefetching helped the situation and made it possible to approach the upper limit imposed by the probability of a control transfer in a particular program. Using the dual branch target buffer, simulations showed that it is possible to achieve performance beyond the $1 / b$ limit. Nevertheless, the fetching performance of a dual branch target buffer is limited to $2 / b$ instructions per cycle.

Our use of models that predict the behavior of fetching performance have proven invaluable in the study of instruction fetching. It enables the production of graphs that clearly show the relationship between different fetching 
options without running hundreds of simulations. They can be helpful in the design of a new superscalar microprocessors to determine which technique will meet the performance objective.

\section{REFERENCES}

[1] B. Calder and D. Grunwald, "Fast and Accurate Instruction Fetch and Branch Prediction," Proc. 21st Ann. Int'l Symp. Computer Architecture, pp. 2-11, Chicago, Apr. 1994.

[2] B. Calder and D. Grunwald, "Reducing Branch Costs Via Branch Alignment," Proc. Sixth Int'l Conf. Architectural Support for Programming Languages and Operating Systems, pp. 242-251, Oct. 1994.

[3] B.G. Calder, "Hardware and Software Mechanisms for Instruction Fetch Prediction," PhD thesis, Univ. of Colorado, Dec. 1995

[4] B. Cmelik and D. Keppel, "Shade: A Fast Instruction-Set Simulator for Execution Profiling," ACM SIGMETRICS, vol. 22, pp. 128137, 1994.

[5] T.M. Conte, K.N. Menezes, P.M. Mills, and B.A. Patel, “Optimization of Instruction Fetch Mechanisms for High Issue Rates," Proc. 22nd Ann. Int'l Symp. Computer Architecture, pp. 333-344, June 1995.

[6] Standard Performance Evaluation Corporation, "SPEC CPU95 Benchmarks," http://www.specbench.org/osg/cpu95, 1 Mar. 1998.

[7] V. Popescu et al., "Metaflow Architecture," IEEE Micro, pp. 10-13, 63-73, June 1991.

[8] G.F. Grohoski, "Machine Organization of the IBM RS/6000 Processor," IBM J. Research and Development, vol. 34, no. 1, pp. 37-58, Jan. 1990.

[9] M. Johnson. Superscalar Microprocessor Design. Englewood Cliffs, N.J.: Prentice Hall, 1991

[10] D.J. Kuck, Y. Muraoka, and S. Chen, "On the Number of Operations Simultaneously Executable in Fortran-Like Programs and Their Resulting Speedup," IEEE Trans. Computers, vol. 21, no. 12, pp. 1,293-1,310, Dec. 1972.

[11] J.K.F. Lee and A.J. Smith, "Branch Prediction Strategies and Branch Target Buffer Design," Computer, vol. 21, no. 7, pp. 6-22, July 1984.

[12] A. Nicolau and J.A. Fisher, "Measuring the Parallelism Available for Very Long Instruction Word Architectures," IEEE Trans. Computers, vol. 33, no. 11, pp. 968-976, Nov. 1984.

[13] E.M. Riseman and C.C. Foster, "The Inhibition of Potential Parallelism by Conditional Jumps," IEEE Trans. Computers, vol. 21, no. 12, pp. 1,405-1,411, Dec. 1972

[14] M.D. Smith, M. Johnson, and M.A. Horowitz, "Limits on Multiple Instruction Issue," Proc. Third Int'l Conf. Architectural Support for Programming Languages and Operating Systems, pp. 290-302, Apr. 1989.

[15] D. Wall, "Limits of Instruction-Level Parallelism," Technical Report 93/6, Digital Equipment Corp., Nov. 1993.

[16] T.-Y. Yeh, D.T. Marr, and Y.N. Patt, "Increasing the Instruction Fetch Rate via Multiple Branch Prediction and a Branch Address Cache," Proc. Seventh ACM Int'l Conf. Supercomputing, pp. 67-76, Tokyo, July 1993.

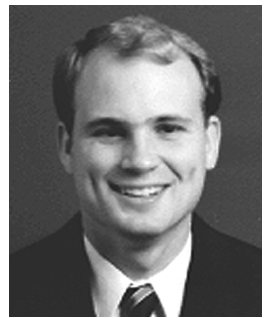

Steven Wallace (M'97) received a BS degree in information and computer science and a BS degree in electrical and computer engineering from the University of California, Irvine, in 1992. $\mathrm{He}$ also received an MS degree and a PhD degree in electrical and computer engineering from the same university in 1993 and 1997, respectively. Recently, he completed his postdoctoral research at the University of California, San Diego. His research interests are computer architecture and VLSI design.

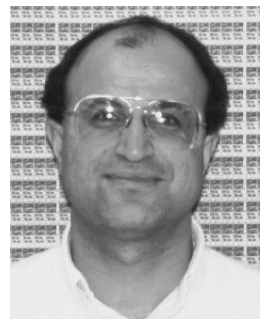

Nader Bagherzadeh (SM'93) received a $\mathrm{PhD}$ degree in computer engineering from the University of Texas at Austin in 1987. From 1980 to 1984, he was with AT\&T Bell Laboratories in Holmdel, New Jersey. Since 1988, he has been with the Department of Electrical and Computer Engineering at the University of California, Irvine. His research interests are in parallel processing, reconfigurable computing, computer architecture, and VLSI design. 